\title{
THERMODYNAMICS OF PHOTON GAS WITH AN INVARIANT ENERGY SCALE
}

\author{
Sudipta Das ${ }^{a}, 1$, Dibakar Roychowdhury ${ }^{b, 2}$ \\ ${ }^{a}$ Physics and Applied Mathematics Unit, Indian Statistical Institute \\ 203 B. T. Road, Kolkata 700108, India \\ ${ }^{b}$ Department of Physics, University of North Bengal \\ Siliguri 734013, West Bengal, India
}

\begin{abstract}
Quantum Gravity framework motivates us to find new theories in which an observer independent finite energy upper bound (preferably Planck Energy) exists. We have studied the modifications in the thermodynamical properties of a photon gas in such a scenario where we have an invariant energy scale. We show that the density of states and the entropy in such a framework are less than the corresponding quantities in Einstein's Special Relativity (SR) theory. This result can be interpreted as a consequence of the deformed Lorentz symmetry present in the particular model we have considered.
\end{abstract}

\footnotetext{
${ }^{1}$ E-mail: sudipta.das_r@isical.ac.in

${ }^{2}$ E-mail: dibakar_nbu@yahoo.co.in
} 


\section{Introduction}

Any description of Quantum Gravity suggests a smallest (but finite) length scale $l$ (or a finite upper bound of energy $\kappa$ ), which of course should be observer independent. The natural candidate for this is the Planck Length (or the Planck Energy). But this proposition obviously contradicts the principles of Einstein's Special Relativity (SR) Theory, as in SR, the length or the mass (or energy) of an object varies for different observers. Thus we need an extension of SR theory where along with the velocity of light, another observerindependent quantity, a fundamental length-scale exists. As a consequence, there must be some modifications of SR theory in the high energy (Planck energy) regime.

As a possible solution, a new theory (DSR Theory) was first proposed by Amelino-Camelia [1]. Another model, perhaps simpler, was given by Amelino-Camelia [2] and by Magueijo and Smolin [3] (for discussion and review, see [4, 5] and references therein). As said earlier, in these theories, there are two invariant quantities, $c$, the velocity of light and $\kappa$, an upper limit of energy. But for consistent inclusion of this second invariant quantity along with the other principles of SR theory, the well known dispersion relation (or mass-shell condition) for a particle

$$
E^{2}-p^{2}=m^{2}
$$

has to be modified as:

$$
E^{2}-p^{2}=m^{2}\left(1-\frac{E}{\kappa}\right)^{2}
$$

Here $E$ and $p$ are respectively the energy and the magnitude of the three-momentum of the particle, $m$ is the mass of the particle and we have taken $c=1$. We refer this model as the Magueijo-Smolin (MS) model.

In earlier work [6], we considered a particular dispersion relation as in [3]. Then we derived an expression for the energy-momentum tensor for a perfect fluid and studied dynamics of the perfect fluid with this modified expression. Due to the presence of the invariant energy scale, our derivation of the energy-momentum tensor was subtle where nonlinear representation of 
Lorentz transformations played an essential role. In this work, we adopt the same scheme and consider (2) as our fundamental equation. Then we go on to study the thermodynamic properties of an ideal photon gas using the methods of conventional statistical mechanics, but generalized to be applicable in a theory where an invariant energy scale is present. We have arranged this paper as follows: In section 2, we discuss about the modified dispersion relation. In the next section we derive the expression for the density of states and the important expression of the partition function. The derivation of the expression for partition function is the most crucial result of our work. In section 4, we go on to study the thermodynamic properties of photon gas using this partition function. In particular, we evaluated analytic expressions for the pressure, equation of state, internal energy, entropy and specific heat of the photon gas. We also show the comparisons between the thermodynamic variables in the MS model and in the usual SR scenario. Further, we see that the density of states as well as the entropy decreases in the MS model as compared to that in the SR framework. This happens due to the deformation in Lorentz symmetry in the theory where an invariant energy scale is present. It is another major result of our work. Finally, we conclude summarizing our results and discuss some of the future prospects in this regard.

\section{Modified Dispersion Relation}

We choose a particular modified dispersion relation as given in [3, 6]

$$
E^{2}-p^{2}=m^{2}\left(1-\frac{E}{\kappa}\right)^{2}
$$

Thermodynamic properties for photon gas with a different dispersion relation has been studied in [7]. Also, thermodynamics of bosons and fermions with another modified dispersion relation and its cosmological and astrophysical implications has been observed in [8, 9]. But these two modified dispersion relations appear from a phenomenological point of view whereas the dispersion relation (2) has a more theoretical motivation which we discuss below 
in some details.

It was shown in [10] that existence of an invariant length scale in the theory is consistent with a non-commutative (NC) phase space ( $\kappa$-Minkowski spacetime) such that the usual canonical Poisson brackets between the phase space variables are modified. Also, the linear Lorentz transformations (LT) are replaced by non-linear $\kappa$-Lorentz transformations $(\kappa$-LT) [11, 10]. But still Lorentz algebra is intact in the theory. As a result, we have the $\kappa$-LT invariant modified dispersion relation (2) as:

$$
\left\{J_{\mu \nu}, \frac{p^{2}}{\left(1-\frac{E}{\kappa}\right)^{2}}\right\}=0 .
$$

The angular momentum $J_{\mu \nu}$ is defined as in [10]

$$
J_{\mu \nu}=x_{\mu} p_{\nu}-x_{\nu} p_{\mu}
$$

where $x$ and $p$ are the phase space variables. Due to the nontrivial expression for the dispersion relation (2), firstly it was supposed that the velocity of photon $c=\frac{d E}{d p}$ have to be energy dependent. But it was shown in [12] that a modified dispersion relation does not necessarily imply a varying (energy dependent) velocity of light. Thus, though the above two models ([7] and [8, 9]) admit a varying speed of light, in case of MS model, for photons $(m=0)$ the dispersion relation (2) is the same as in SR theory. Also the speed of light $c$ is an invariant quantity in the MS model [3, 10, 6]. Thus the MS model considered in [3, 10] has a more theoretical motivation and it can be developed starting from the NC phase space variables [10] whereas the models considered in [7, 8, 9] are phenomenological in nature and as far as we know, there is no fundamental phase space structures to describe these models. Another interesting fact is that both the models described in [7] and in [8, 9] have no finite upper bound of energy of the photons though they have a momentum upper bound. But, as stated earlier, in the MS case, though the dispersion relation for the photons is unchanged, there is a finite upper bound of photon energy which is the Planck energy $\kappa$. One can readily check that this is an invariant quantity by using the $\kappa$-Lorentz transformation law for the energy [11, 10]. 
One more thing must be clarified here. In case of the models ([7] and [8, 9]), clearly the Lorentz symmetry was broken and as a result, the number of microstates and hence the entropy increases as compared to the Lorentz symmetric SR theory. On the other hand, we are dealing with a different scenario where the Lorentz symmetry is not broken as Lorentz algebra between the phase space variables is intact. In fact, the framework we describe here still satisfies the basic postulates of Einstein's SR theory; moreover it possesses another observer-independent quantity [4]. Thus it seems that Lorentz symmetry is further restricted

in this MS model. As a result of this, we expect to have a less number of microstates and less entropy in the Ms model. As we will show later in our explicit calculations, this expected result is correct.

As we have said earlier, the modified dispersion relation (21) in case of the photons (massless particles) does not change from the usual SR scenario. Thus, for the photons, the dispersion relation now becomes

$$
p=E
$$

\section{Partition Function for Photon Gas}

To study the thermodynamic behavior of photon gas, we have to find out an expression for the partition function first, as it relates the microscopic properties with the thermodynamic (macroscopic) behavior of a physical system [13, 14], which we do in this section.

\subsection{Number of states:}

We consider a box containing photon gas. Following the standard procedure as given in [13, 14], we consider a continuous spectrum of momentum instead of quantizing it. The 
number of microstates available to the system $\left(\sum\right)$ in the position range from $r$ to $r+d r$ and in the momentum range from $p$ to $p+d p$ is given by [13, 14]

$$
\sum=\frac{1}{h^{3}} \iint d^{3} \vec{r} d^{3} \vec{p}
$$

where $h$ is the phase space volume of a single lattice and

$$
\iint d^{3} \vec{r} d^{3} \vec{p}
$$

is the total phase space volume available to the system.

It should be mentioned here that in case of SR theory, the quantities $E d^{3} x$ and $\frac{d^{3} p}{E}$ are invariant under the Lorentz transformations and hence the phase space volume element $d^{3} x d^{3} p$ is a Lorentz invariant quantity [15]. The nonlinear $\kappa$-Lorentz transformations [11, 10] are explicitly given by:

$$
\begin{gathered}
t^{\prime}=\alpha \gamma(t-v x), \quad x^{\prime}=\alpha \gamma(x-v t), \quad y^{\prime}=\alpha y \quad, \quad z^{\prime}=\alpha z \\
E^{\prime}=\frac{\gamma\left(E-v p_{x}\right)}{\alpha}, \quad p_{x}^{\prime}=\frac{\gamma\left(p_{x}-v E\right)}{\alpha}, \quad p_{y}^{\prime}=\frac{p_{y}}{\alpha}, \quad p_{z}^{\prime}=\frac{p_{z}}{\alpha} .
\end{gathered}
$$

The prime over a quantity denotes the corresponding quantity in the boosted frame and $\alpha=1+\frac{1}{\kappa}\left((\gamma-1) E-v \gamma p_{x}\right)$. We have considered the three-momentum to be of the usual form: $\vec{p}=(v E, 0,0)$ and $\gamma=\frac{1}{\sqrt{1-v^{2}}}$ where $v$ is the velocity of the boosted frame. In case of our model, the phase space volume element $d^{3} x d^{3} p$ is invariant under the $\kappa$-Lorentz transformations (3) as:

$$
d^{3} x^{\prime} d^{3} p^{\prime}=\alpha^{3} \gamma d^{3} x \frac{\gamma}{\alpha^{3}}\left(1-\frac{v p_{x}}{E}\right) d^{3} p=d^{3} x d^{3} p
$$

It is interesting to note that the factor $\alpha$ arising from the nonlinear $\kappa$-Lorentz transformation finally cancels out in (7). Strictly speaking, to derive (7) we should consider the effect coming from variation of $\alpha$. But we have omitted the term $d \alpha$ in (7) as it is a dynamical effect and may not be relevant for the free particle case as considered here.

If the volume of the box is considered to be $V$, the number of microstates can be written in the following form using the spherical polar coordinates [13, 14]

$$
\sum=\frac{4 \pi V}{h^{3}} \int_{0}^{\infty} E^{2} d E .
$$


We used the dispersion relation $p=E$ to change the integration variable to $E$. Then considering the fact that we have an finite upper limit of energy $(\kappa)$, we obtain the number of microstates:

$$
\tilde{\sum}=\frac{4 \pi V}{h^{3}} \int_{0}^{\kappa} E^{2} d E
$$

where $\sim$ on a quantity represents the corresponding quantity in the model we have considered. It is obvious from the expressions (8) and (9) that the available number of microstates to the system is less than that in the SR theory, as the energy spectrum of a particle in SR theory can go all the way up till infinity. This result agrees with our expectation stated earlier.

\subsection{Partition function:}

It is very crucial to get an expression for the partition function as all the thermodynamic properties can be thoroughly studied using the knowledge about the partition function. The single particle partition function $Z_{1}(T, V)$ is defined as [14]

$$
Z_{1}(T, V)=\frac{4 \pi V}{h^{3}} \int_{0}^{\infty} p^{2} e^{-\beta E} d p
$$

where $\beta=\frac{1}{k_{B} T}, k_{B}$ is the Boltzman constant and $T$ is the temperature of the particle.

For the MS model, the single particle partition function $\tilde{Z}_{1}(T, V)$ is defined as

$$
\tilde{Z}_{1}(T, V)=\frac{4 \pi V}{h^{3}} \int_{0}^{\kappa} p^{2} e^{-\beta E} d p .
$$

In the limit $\kappa \rightarrow \infty$, we should get back normal SR theory results.

It should be noted that in the MS model which we have considered, the photon dispersion relation is not modified at all. But still there is modification in the partition function (11) due to the presence of an energy upper bound of particles $(\kappa)$ in the theory. So the upper limit of integration is $\kappa$ in (11) whereas in the normal SR theory expression (10), the upper limit of integration is $\infty$ since there is no upper bound of energy in the SR theory. In all the models [7, 8, 9], though the upper limit of energy is infinity as in SR theory, these models 
differ due to the different dispersion relations.

Using the dispersion relation for photons $(E=p)$ and using the standard table and formulae for integrals [16], we finally have an analytical expression of the single particle partition function

$$
\tilde{Z}_{1}(T, V)=\frac{4 \pi V}{h^{3}} \int_{0}^{\kappa} E^{2} e^{-\beta E} d E=\frac{4 \pi V}{h^{3}}\left[\frac{2}{\beta^{3}}-\frac{e^{-\beta \kappa}}{\beta^{3}}(2+\beta \kappa(2+\beta \kappa))\right] .
$$

Thus the partition function for a $N$-particle system $\tilde{Z}_{N}(T, V)$ is given by

$$
\tilde{Z}_{N}(T, V)=\frac{1}{N !}\left[\tilde{Z}_{1}(T, V)\right]^{N}
$$

where we have considered classical (Maxwell-Boltzman) statistics along with the Gibb's factor. As we get the expression for the partition function, now we go on to study various thermodynamic properties of the photon gas in our model. It should be noted that as $\kappa \rightarrow \infty$, this partition function coincides with the partition function in SR theory and thus all of our results coincides with the usual SR case in this limit.

\section{Thermodynamic Properties of Photon Gas}

With the expression for the partition function in our hand, now we go on to study various thermodynamic properties of photon gas in a theory where an observer-independent fundamental energy scale is present.

\subsection{Free energy:}

We use Stirling's formula for $\ln [N !]$ [14]

$$
\ln [N !] \approx N \ln [N]-N
$$

in the expression for partition function (13) to obtain the free energy $\tilde{F}$ of the system

$$
\tilde{F}=-k_{B} T \ln \left[\tilde{Z}_{N}(T, V)\right]
$$




$$
=-N k_{B} T\left[1+\ln \left[\frac{4 \pi V}{N}\left(\frac{k_{B} T}{h}\right)^{3}\left\{2-e^{-\frac{\kappa}{k_{B} T}}\left(2+\frac{\kappa}{k_{B} T}\left(2+\frac{\kappa}{k_{B} T}\right)\right)\right\}\right] .\right.
$$

In the limit $\kappa \rightarrow \infty$, the terms containing $\kappa$ vanishes and we get back normal SR theory result:

$$
F=N k_{B} T
$$

\subsection{Pressure:}

From the expression for free energy (3) we can readily obtain the pressure $\tilde{P}$ of photon gas in our considered model as [14]

$$
\tilde{P}=-\left(\frac{\partial F}{\partial V}\right)_{T, N}=\frac{N k_{B} T}{V}
$$

Thus, we have the same equation of state

$$
P V=N k_{B} T
$$

as in SR theory.

\subsection{Entropy:}

As we have the expression for free energy (3), also we can evaluate the entropy $\tilde{S}$ of the system from the following relation [14]

$$
\tilde{S}=-\left(\frac{\partial F}{\partial T}\right)_{V, N}
$$

The expression for entropy takes the following form

$$
\begin{gathered}
\tilde{S}=N k\left[4+\ln \left[\frac{4 \pi V}{N}\left(\frac{k_{B} T}{h}\right)^{3}\left\{2-e^{-\frac{\kappa}{k_{B} T}}\left(2+\frac{\kappa}{k_{B} T}\left(2+\frac{\kappa}{k_{B} T}\right)\right)\right\}\right]\right. \\
\left.-\frac{\kappa^{3}}{2 k_{B}^{3} T^{3} e^{\frac{\kappa}{k_{B} T}}-\left(2 k_{B}^{3} T^{3}+2 k_{B}^{2} T^{2} \kappa+k_{B} T \kappa^{2}\right)}\right] .
\end{gathered}
$$


The terms containing $\kappa$ are the modifications from the SR theory expression of entropy [14]. As in the earlier expressions, in the limit $\kappa \rightarrow \infty$ the terms containing $\kappa$ vanish and we get back the SR theory result:

$$
S=N k_{B}\left[4+\ln \left[\frac{8 \pi V}{N}\left(\frac{k_{B} T}{h}\right)^{3}\right]\right] .
$$

We plot the entropy $S$ against $T$ both for the model we considered and for SR theory to study the deviation of entropy in the two models.

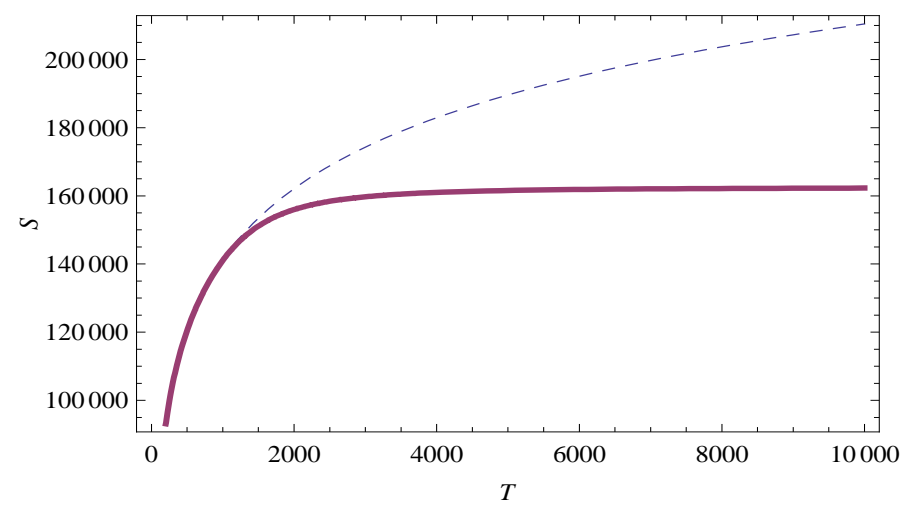

Figure 1: Plot of entropy of photon $S$ against temperature $T$ for both in the SR theory and in our case; the dashed line corresponds to the SR theory result and the thick line represents the corresponding quantity in our result. We have used the Planck units and the corresponding parameters take the following values $\kappa=10000, k_{B}=1, N=10000, V=.01, h=1$ in this plot as well as in all other plots in the paper. In this scale, $T=10000$ is the Planck temperature.

In Figure 1, we have plotted entropy against temperature for both the case of our invariant energy scale scenario and normal SR theory. It is clearly observable from the plot that the entropy grows at a much slower rate in case of our result than in the SR theory and as temperature increases, the entropy in our considered model deviates more from the entropy in the SR theory. This result matches with our earlier expectation considering the underlying symmetry of the theory that the entropy in the MS model should be less than the entropy in SR theory. 
It is well known that the total number of microstates available to a system is a direct measure of the entropy for that system. Therefore our result merely reflects the fact that due to the existence of an energy upper bound $\kappa$, the number of microstates gradually saturates to some finite value near Planck scale.

\subsection{Internal energy:}

We expect modification in the expression of the internal energy $U$ for photon gas in the MS model as the expression of entropy is modified and internal energy is related to the entropy as follows:

$$
U=F+T S
$$

In the usual SR scenario, the explicit expression for internal energy is given by

$$
U=3 N k_{B} T
$$

But in the MS scenario we considered, the expression for internal energy $(\tilde{U})$ of photon gas takes the following form

$$
\tilde{U}=N k_{B} T\left[3-\frac{\kappa^{3} e^{-\frac{\kappa}{k_{B} T}}}{2 k_{B}^{3} T^{3}-e^{-\frac{\kappa}{k_{B} T}}\left(2 k_{B}^{3} T^{3}+2 \kappa k_{B}^{2} T^{2}+\kappa^{2} k_{B} T\right)}\right] .
$$

It is easy to see from the expression of internal energy (19) that we get back the usual SR theory expression in the limit $\kappa \rightarrow \infty$. As in the case of entropy, here we also plot internal energy against temperature for both the SR and MS case.

In Figure 2, we plotted internal energy of photon gas against its temperature for both the case of MS model and SR theory. The expression in SR theory (18) tells us that internal energy depends linearly on the temperature and this is supported from the plot. But from the expression of internal energy in the MS model (19) it is clearly observed that the relation of internal energy with temperature is not linear at all. Also one can easily check that the value of internal energy (for a particular temperature) in the MS model (19) is always less than its value (for the same temperature) in the SR theory (18). This is very clear from the 


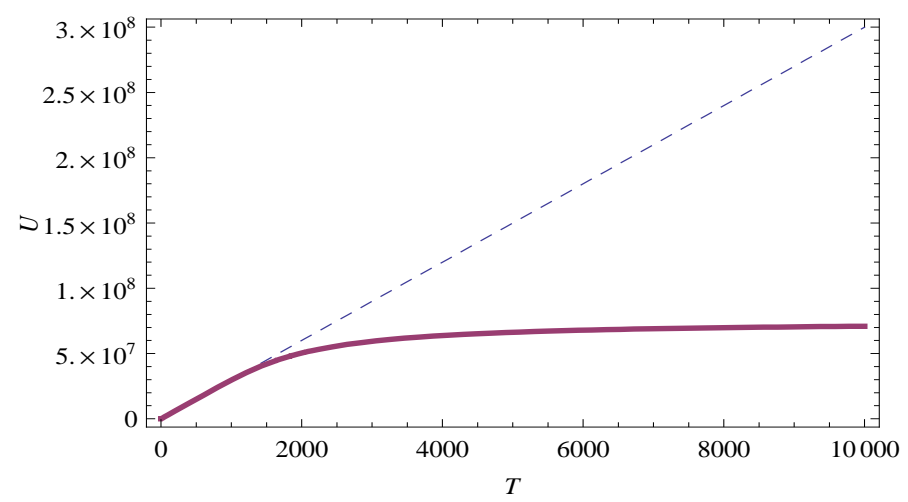

Figure 2: Plot of internal energy of photon $U$ against temperature $T$ for both in the $S R$ theory and MS scenario; the dashed line corresponds to the SR theory result and the thick line represents the quantity in the MS model we considered here.

plot as the curve for the MS model always lies below the straight line which corresponds the SR theory result.

Since the internal energy $U$ of photon gas becomes saturated after a certain temperature

in case of the MS model, it is tempting to point out that probably our results are moving towards the right direction related to the "Soccer Ball Problem" that plagues multi-particle description in the framework of DSR. The problem lies in the fact that if we apply linear addition rule for momenta/energies of many sub-Planck energy particles we may end up with a multi-particle state, such as a soccer ball, whose total energy is greater than the Planck energy which is forbidden in the DSR theory. For further discussion about the "Soccer Ball Problem" see [17].

\subsection{Pressure-energy density relation:}

Though internal energy $U$ of a physical system is not directly measurable, still we can detect the effect of it through other thermodynamic quantities, such as the relation between pressure $P$ and energy density $\rho$ of that system. Energy density of a system $\rho$ is defined as

$$
\rho=U / V
$$


where $U$ is the internal energy of the system and $V$ is the volume occupied by the system. As the expression for internal energy $U$ is modified in the MS model, we also expect modifications in the expression for the energy density $\rho$. The modified relation between pressure $\tilde{P}$ and energy density $\tilde{\rho}$ is given by:

$$
\tilde{P}=\frac{1}{3} \tilde{\rho}+\frac{1}{3} \frac{N k_{B} T \kappa^{3} e^{-\frac{\kappa}{k_{B} T}}}{2 V k_{B}^{3} T^{3}-V e^{-\frac{\kappa}{k_{B} T}}\left(2 k_{B}^{3} T^{3}+2 \kappa k_{B}^{2} T^{2}+\kappa^{2} k_{B} T\right)} .
$$

For $\kappa \rightarrow \infty$, we get back the usual pressure-energy density relation in SR theory:

$$
P=\frac{1}{3} \rho
$$

It should be pointed out that in our earlier work [6], it was shown that in the ultra-relativistic

regime (for photons), the relation $P=\frac{1}{3} \rho$ remains unaffected. But here we have a modification in this pressure-energy density relation (20). In [6], we obtained the result considering some simplified assumptions. But in this work, we start with the partition function and apply the methods of Statistical Mechanics (which naturally deals with multi-particle systems). So we do not really have to consider any strong assumptions here.

\subsection{Specific heat:}

There is another thermodynamic parameter, specific heat $\left(C_{V}\right)$, through which we can observe the modifications in the expression for internal energy. Specific heat $C_{V}$ is defined as

$$
C_{V}=\left(\frac{\partial U}{\partial T}\right)_{V}
$$

For the MS model we considered here, explicit calculation yields the following result

$$
\tilde{C}_{V}=3 N k_{B}-\frac{2 N k_{B} \kappa^{3}\left(k_{B}^{2} T^{2} \kappa\left(1+e^{\frac{\kappa}{k_{B} T}}\right)+2 k_{B}^{3} T^{3}\left(1-e^{\frac{\kappa}{k_{B} T}}\right)\right)}{\left(2 k_{B}^{3} T^{3}\left(e^{\frac{\kappa}{k_{B} T}}-1\right)-2 k_{B}^{2} T^{2} \kappa-k_{B} T \kappa^{2}\right)^{2}} .
$$

After doing a bit of algebra, one can check from the above expression (21) that specific heat calculated from the MS model is always less than the value calculated from usual SR theory. Also, when $\kappa \rightarrow \infty$, we obtain

$$
C_{V}=3 N k_{B}
$$


which is the usual specific heat for photon as calculated in SR theory.

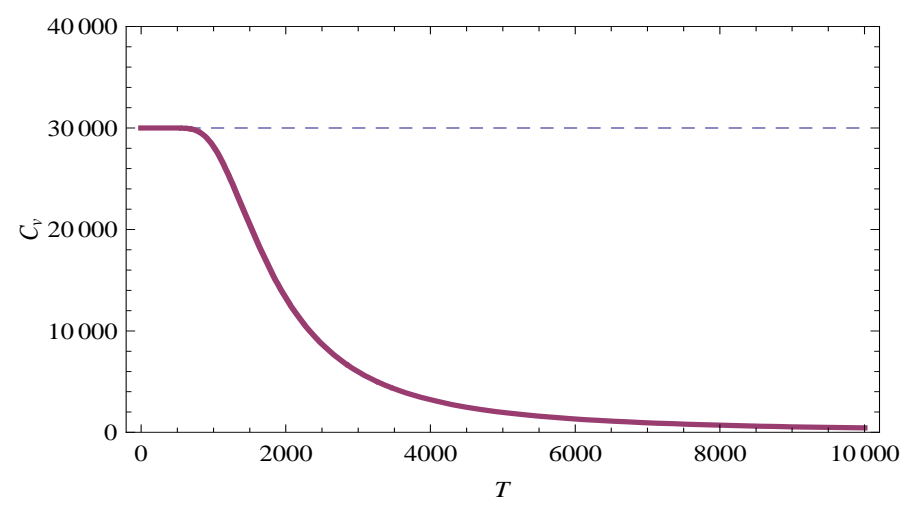

Figure 3: Plot of specific heat of photon $C_{V}$ against temperature $T$ for both in the $S R$ theory and MS scenario; the dashed line corresponds to the SR theory result and the thick line represents the quantity in the $M S$ model we considered here.

In Figure 3, we have plotted the specific heat $C_{V}$ against temperature $T$. It is clear from the plot that in the case of the MS model, the specific heat $C_{V}$ asymptotically decreases to zero suggesting that photon gas has reached its temperature ceiling which is the Planck temperature.

\section{Conclusion and Future Prospects}

We consider the modified dispersion relation as given in the MS model [3]. We explicitly show that for photons, the number of microstates available to a macrostate is less in the MS model than in the usual SR scenario. We stress that it happens since Lorentz symmetry is not broken in this model. But due do the presence of an invariant energy upper bound in this theory, microstates can avail energies only up to a finite cut-off whereas in SR theory, microstates can attain energies up to infinity. Thus, quite naturally, the number of microstates in this MS model is less than that in SR theory.

The most significant result of our work is the derivation of $N$-particle partition function in the MS model. Due to the presence of the deformed dispersion relation, this task becomes 
highly non-trivial. However, for photons, we find out an analytic expression for the partition function. Once we have the partition function in our hand, we evaluate other various thermodynamic parameters of photon gas such as the free energy, pressure, entropy, internal energy, specific heat for the MS model and compare them with the known results of SR theory.

As a consequence of deformed Lorentz symmetry, the entropy in the MS model is also less than that in the SR scenario. We show this behavior analytically and graphically. Also the internal energy is modified in case of the MS model and as a consequence the expression for the specific heat is also modified.

Though highly non-trivial, one can similarly study the behavior of an ideal gas using this modified dispersion relation. Also one can study behavior of fermion gas in this MS model. There might be some modifications in the Fermi energy level which can modify the Chandrasekhar mass limit for the white dwarf stars [9]. Thus astrophysical phenomena in an MS framework is another issue remains to be addressed.

Further, as we have the expression for energy-momentum tensor, one can study the cosmological aspects of MS model using the Friedmann equations. But this requires idea about the geometry sector (precisely the metric $g_{\mu \nu}$ and hence Einstein tensor $G_{\mu \nu}$ ) which is till unknown in the context of MS model. This still remains another open issue to be further studied.

It is noteworthy to mention here that "bouncing" loop quantum cosmology theories (for example see [18] and references therein) entails some modifications to the geometry of spacetime which in turn effectively puts a bound on the curvature avoiding the big bang singularity. However, for these "bouncing" models, the perturbation technique cannot be done as at the point of curvature saturation, the energy density of the cosmic fluid diverges. So it is unclear how to construct the matter part of Einstein equation. One alternative to avoid the big bang singularity is the inflation theory where the perturbation method can also be applied. On the other hand, in our model, the energy density of the cosmic fluid 
saturates to the Planck energy which is a finite real quantity. Possibly a combination of the model considered in this paper along with the "bouncing" loop quantum cosmology can successfully describe a situation where big bang singularity can be avoided.

\section{Acknowledgements}

We would like to thank Prof. Subir Ghosh for helpful discussions.

\section{References}

[1] G. Amelino-Camelia, Nature 41834 (2002); Phys. Lett. B 510 (2001) 255; Int. J. Mod. Phys. A 11 (2002) 35.

[2] G. Amelino-Camelia, Int. J. Mod. Phys. D 11 (2002) 35 [arXiv: gr-qc/0012051].

[3] J. Magueijo and L. Smolin, Phys. Rev. Lett. 88 (2002) 190403; Phys. Rev. D 67 (2003) 044017.

[4] G. Amelino-Camelia, Recent Developments in Theoretical Physics (Statistical Science and Interdisciplinary Research-Vol. 9), edited by Subir Ghosh and Guruprasad Kar, World Scientific, Singapore (2010).

[5] J. Kowalski-Glikman, Lect. Notes Phys. 669 (2005) 131 [arXiv: hep-th/0405273].

[6] S. Das, S. Ghosh and D. Roychowdhury, Phys. Rev. D 80 (2009) 125036 [arXiv: 0908.0413].

[7] A. Camacho and A. Macius, Gen. Rel. Grav. 39 (2007) 1175 [arXiv: gr-qc/0702150].

[8] S. Alexander and J. Magueijo, Proceedings of the XIIIrd Rencontres de Blois (2004) 281 [arXiv: hep-th/0104093].

[9] O. Bertolami and C. A. D. Zarro, Phys. Rev. D 81 (2010) 025005 [arXiv: 0908.4196]; M. Gregg and S. A. Major, Int. J. Mod. Phys. D 18, 971 (2009) [arXiv: 0806.3496]. 
[10] S. Ghosh and P. Pal, Phys. Rev. D 75 (2007) 105021 [arXiv: hep-th/0702159].

[11] N. R. Bruno, G. Amelino-Camelia and J. Kowalski-Glikman, Phys. Lett. B 522 (2001) 133 [arXiv: hep-th/0107039].

[12] S. Hossenfelder, Class. Quant. Grav. 23 (2006) 1815 [arXiv: hep-th/0510245].

[13] R. K. Pathria, Statistical Mechanics, Butterworth-Heinemann, Oxford (1996).

[14] W. Greiner and N. Stocker, Thermodynamics and Statistical Mechanics, SpringerVerlag, New York (1995).

[15] C. W. Misner, K. S. Thorne and J. A. Wheeler, Gravitation, W. H. Freeman and Co., New York (1973).

[16] I. S. Gradshteyn and I. M. Ryzhik, Table of Integrals, Series and Products (Vol. 1), Academic Press, Inc., New York (1994).

[17] J. Magueijo, Phys. Rev. D 73 (2006) 124020 [arXiv: gr-qc/0603073]; S. Hossenfelder, Phys. Rev. D 75 (2007) 105005 [arXiv: hep-th/0702016].

[18] M. Bojowald [arXiv: 1002.2618]. 